\title{
The mortality benefit of carvedilol versus bisoprolol in patients with heart failure with reduced ejection fraction
}

Ki Hong Choi ${ }^{1}$, Ga Yeon Lee ${ }^{1}$, Jin-Oh Choi ${ }^{1}$, Eun-Seok Jeon ${ }^{1}$, Hae-Young Lee $^{2}$, Sang Eun Lee ${ }^{3}$, Jae-Joong Kim³ ${ }^{3}$ Shung Chull Chae ${ }^{4}$, Sang Hong Baek ${ }^{5}$, Seok-Min Kang ${ }^{6}$, Dong-Ju Choi ${ }^{7}$, Byung-Su Yoo ${ }^{8}$, Kye Hun $\mathrm{Kim}^{9}$, Myeong-Chan $\mathrm{Cho}^{10}$, Hyun-Young Park ${ }^{11}$, and Byung-Hee $\mathrm{Oh}^{2}$

\begin{abstract}
${ }^{1}$ Department of Medicine, Sungkyunkwan University School of Medicine, Seoul; ${ }^{2}$ Department of Internal Medicine, Seoul National University Hospital, Seoul; ${ }^{3}$ Department of Internal Medicine, University of Ulsan College of Medicine, Seoul; ${ }^{4}$ Department of Internal Medicine, School of Medicine, Kyungpook National University, Daegu; ${ }^{5}$ Department of Internal Medicine, College of Medicine, The Catholic University of Korea, Seoul; ${ }^{6}$ Department of Internal Medicine, Yonsei University College of Medicine, Seoul; ${ }^{7}$ Department of Internal Medicine, Seoul National University Bundang Hospital, Seongnam; ${ }^{8}$ Department of Internal Medicine, Yonsei University Wonju College of Medicine, Wonju; ${ }^{9}$ Heart Research Center of Chonnam National University, Gwangju; ${ }^{10}$ Department of Internal Medicine, Chungbuk National University College of Medicine, Cheongju; ${ }^{11}$ National Institute of Health (NIH), Osong, Korea
\end{abstract}

Background/Aims: It is unknown whether different $\beta$-blockers (BBs) have variable effects on long-term survival of patients with heart failure with reduced ejection fraction (HFrEF). This study compares the effects of two BBs, carvedilol and bisoprolol, on survival in patients with HFrEF.

Methods: The Korean Acute Heart Failure (KorAHF) registry is a prospective multicenter cohort that includes 5,625 patients who were hospitalized for acute heart failure (AHF). We selected 3,016 patients with HFrEF and divided this study population into two groups: $B B$ at discharge $(n=1,707)$ or no BB at discharge $(n$ $=1,309$ ). Among patients with BB at discharge, subgroups were formed based on carvedilol prescription $(n=831)$, or bisoprolol prescription $(n=553)$. Propensity score matching analysis was performed.

Results: Among patients who were prescribed a BB at discharge, $60.5 \%$ received carvedilol and $32.7 \%$ received bisoprolol. There was a significant reduction in allcause mortality in those patients with HFrEF prescribed a BB at discharge compared to those who were not (BB vs. no BB, $26.1 \%$ vs. $40.8 \%$; hazard ratio [HR], 0.59; $95 \%$ confidence interval [CI], 0.52 to $0.67 ; p<0.001$ ). However, there was no significant difference in the rate of all-cause mortality between those receiving different types of BB (carvedilol vs. bisoprolol, $27.5 \%$ vs. $23.5 \%$; HR, 1.21; 95\% CI, 0.99 to 1.47; $p=0.07)$. Similar results were observed after propensity score matching analysis (508 pairs, $26.2 \%$ vs. $23.8 \%$; HR, 1.10; $95 \%$ CI, 0.86 to $1.40 ; p=0.47$ ).

Conclusions: In the treatment of AHF with reduced EF after hospitalization, mortality benefits of carvedilol and bisoprolol were comparable.

Keywords: Beta-blocker; Heart failure with reduced ejection fraction; Carvedilol; Bisoprolol
Received: January 6, 2018 Revised : March 14, 2018 Accepted: April 2, 2018
Correspondence to Jin-Oh Choi, M.D.

Division of Cardiology, Department of Medicine, Cardiac and Vascular Center, Samsung Medical Center, Sungkyunkwan University School of Medicine, 81 Irwon-ro, Gangnam-gu, Seoul 06351, Korea

Tel: +82-2-3410-3419, Fax: +82-2-3410-3849, E-mail: choijean5@gmail.com 


\section{INTRODUCTION}

The clinical benefits of $\beta$-blockers (BBs) are well established in patients with heart failure with reduced ejection fraction (HFrEF). Therefore, current guidelines recommend $\mathrm{BBs}$ in treatment of patients with stable HFrEF to reduce the risk of heart failure (HF) hospitalization and death $[1,2]$. Among the many types and classifications of BBs, three drugs (bisoprolol, sustained release metoprolol succinate, and carvedilol) have been shown to be effective in reducing the risk of death in patients with HFrEF [3-6]. However, most randomized controlled trials that have evaluated the effect of BBs in patients with HFrEF have compared BBs to placebo. Therefore, it is unknown whether the beneficial effects in HF are different among the well-established BBs. Carvedilol has $\beta 1-, \beta 2-$, and $\alpha 1$-receptor blocking effects, unlike bisoprolol and metoprolol, which are $\beta 1$-selective receptor blockers [7]. One head-to-head comparison trial between carvedilol and metoprolol tartrate found that carvedilol increased survival in patients with chronic HF [8]. However, there are conflicting results regarding clinical outcomes according to the use of BBs of different classes based on registry or meta-analysis data. In particular, there are limited data available to directly compare the efficacy of carvedilol versus bisoprolol [9-13].

Therefore, this study sought to compare the mortality effects of carvedilol and bisoprolol prescribed at discharge to patients hospitalized for acute heart failure (AHF) using multicenter prospective registry data in Korea.

\section{METHODS}

\section{Study population and data collection}

Details on the study design of the Korean Acute Heart Failure (KorAHF) registry have been documented previously (Clinicaltrials.gov, NCTo1389843) [14,15]. Briefly, 5,625 consecutive patients from 10 tertiary hospitals in Korea who were hospitalized for AHF were enrolled between March 2011 and February 2014. Patients were included if they had signs or symptoms of HF such as lung congestion, objective findings of left ventricular systolic dysfunction, or structural heart disease. Demo- graphic and clinical information, including medical history, signs, symptoms, laboratory results, electrocardiogram, echocardiography, medications, hospital course, and outcomes, was obtained prospectively at the time of admission, discharge, and during the follow-up period (30-day, 90-day, 180-day, 1- to 5-year annually). Mortality data from patients lost to follow-up were collected from the National Insurance or National Death Records. Fig. 1 presents the distribution of the study population. The study protocol was approved by the Institutional Review Board (IRB No. 1102-072-352) of each participating hospital. The written informed consent was waived, and from the third year of enrollment, the written consents were received from survivals for further follow-up.

\section{Definitions and outcomes}

HFrEF was defined as left ventricular ejection fraction $(\mathrm{LVEF})<40 \%$ according to recent guidelines [1]. LVEF was assessed using the biplane Simpson technique, M-mode, or visual estimation [16]. Renal function was assessed based on the estimated glomerular filtration

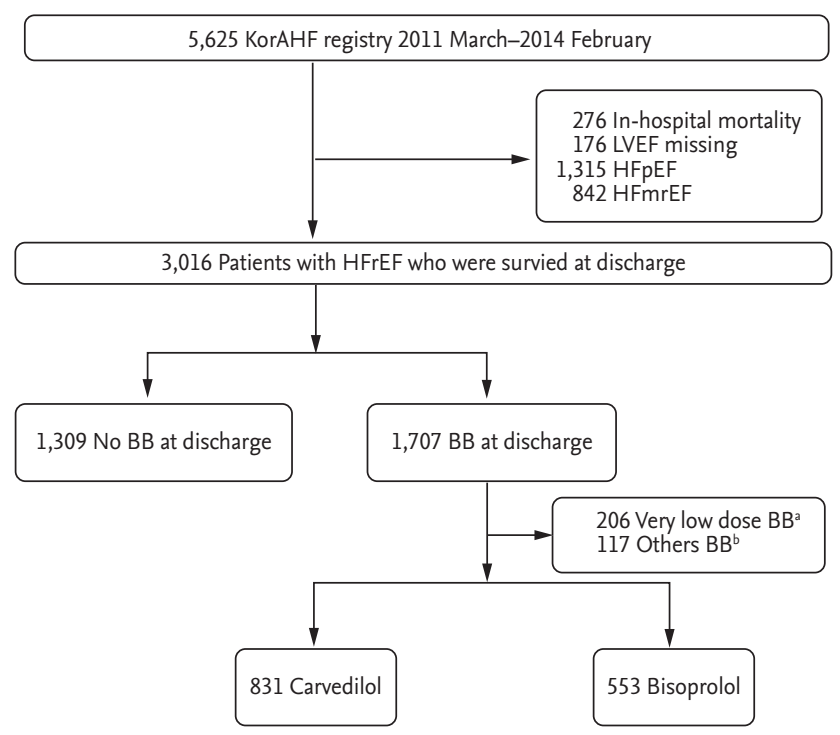

Figure 1. Study flow. KorAHF, Korean Acute Heart Failure; LVEF, left ventricular ejection fraction; HFpEF, heart failure with preserved ejection fraction; HFmrEF, heart failure with mid-range ejection fraction; HFrEF, heart failure with reduced ejection fraction; $\mathrm{BB}, \beta$-blocker. ${ }^{\text {a}}$ Very low dose BB was defined as a standardized dose of carvedilol $\leq 3.125 \mathrm{mg}$, ${ }^{\mathrm{b}}$ Other BBs included nebivolol, metoprolol, betaxolol, celiprolol, atenolol, propranolol, amosulalol, bevantolol, and sotalol. 
Table 1. Baseline clinical and laboratory characteristics

\begin{tabular}{|c|c|c|c|c|c|c|c|c|}
\hline \multirow[b]{2}{*}{ Characteristic } & \multicolumn{4}{|c|}{ Overall population } & \multicolumn{4}{|c|}{ Propensity matched population } \\
\hline & $\begin{array}{c}\text { Bisoprolol } \\
(\mathrm{n}=553)\end{array}$ & $\begin{array}{l}\text { Carvedilol } \\
(\mathrm{n}=831)\end{array}$ & $p$ value & SMD & $\begin{array}{l}\text { Bisoprolol } \\
(\mathrm{n}=508)\end{array}$ & $\begin{array}{l}\text { Carvedilol } \\
(\mathrm{n}=508)\end{array}$ & $p$ value & SMD \\
\hline Age, yr & $65.95 \pm 15.0$ & $64 \cdot 31 \pm 14 \cdot 5$ & 0.43 & $4 \cdot 3$ & $64.94 \pm 14.9$ & $63.99 \pm 14.4$ & 0.30 & 6.3 \\
\hline Male sex & $321(58.1)$ & $526(63 \cdot 3)$ & 0.06 & -10.8 & $303(59 \cdot 7)$ & $307(60.4)$ & 0.85 & -1.6 \\
\hline Current smoker & $124(22.4)$ & $210(25 \cdot 3)$ & 0.25 & -6.7 & $119(23.4)$ & $127(25 \cdot 0)$ & 0.61 & -3.8 \\
\hline $\mathrm{BMI}, \mathrm{kg} / \mathrm{m}^{2}$ & $23.4 \pm 3.8$ & $23.7 \pm 3.9$ & 0.14 & -8.1 & $23.4 \pm 3.8$ & $23.5 \pm 3.7$ & 0.63 & -3.0 \\
\hline Hypertension & $293(53.0)$ & $491(59.1)$ & $0.03^{\mathrm{a}}$ & $-12 \cdot 3$ & $276(54 \cdot 3)$ & $269(53.0)$ & 0.71 & 2.8 \\
\hline Diabetes mellitus & $217(39.2)$ & $318(38.3)$ & 0.76 & 2.0 & $201(39 \cdot 6)$ & $196(38.6)$ & 0.80 & 2.0 \\
\hline $\begin{array}{l}\text { Chronic kidney } \\
\text { disease }\end{array}$ & $67(12.1)$ & $116(14.0)$ & 0.36 & $-5 \cdot 5$ & $64(12.6)$ & $64(12.6)$ & $>0.99$ & o \\
\hline Pulmonary disease & $49(8.7)$ & $58(7 \cdot 0)$ & 0.24 & $7 \cdot 0$ & $44(8.7)$ & $39(7.7)$ & 0.65 & 3.5 \\
\hline Prior HF admission & $136(24 \cdot 6)$ & $254(30.6)$ & $0.02^{\mathrm{a}}$ & $-13 \cdot 4$ & $129(25 \cdot 4)$ & $132(26.0)$ & 0.89 & -1.4 \\
\hline De novo HF & $336(60.8)$ & $453(54 \cdot 5)$ & $0.03^{\mathrm{a}}$ & -12.7 & $290(57.1)$ & $271(48.3)$ & 0.26 & $-7 \cdot 5$ \\
\hline ICMP & $205(37.1)$ & $342(41.2)$ & 0.14 & -8.4 & $195(38.4)$ & $194(38.2)$ & $>0.99$ & 0.4 \\
\hline Atrial fibrillation & $115(20.8)$ & $182(21.9)$ & 0.67 & -2.7 & $105(20.7)$ & $109(21.5)$ & 0.82 & -2.0 \\
\hline $\mathrm{NYHA} \geq 3$ & $469(84.8)$ & $706(85.0)$ & $>0.99$ & -0.4 & $431(84.8)$ & $433(85 \cdot 2)$ & 0.93 & -1.1 \\
\hline $\mathrm{SBP}, \mathrm{mmHg}$ & $130.3 \pm 26.8$ & $133.0 \pm 31.1$ & 0.08 & $-9 \cdot 4$ & $130.8 \pm 27.1$ & $130.7 \pm 30.0$ & 0.98 & 0.2 \\
\hline $\mathrm{DBP}, \mathrm{mmHg}$ & $82.4 \pm 18.7$ & $82.3 \pm 19.4$ & 0.91 & 0.6 & $82.2 \pm 18.6$ & $82.0 \pm 19.1$ & 0.91 & 0.7 \\
\hline Pulse rate, beats/min & $94.5 \pm 23.5$ & $96.6 \pm 25.2$ & 0.13 & -8.5 & $94.8 \pm 23.1$ & $94.1 \pm 25.1$ & 0.64 & 3.0 \\
\hline LVEF, \% & $27.1 \pm 7.1$ & $26.3 \pm 7.5$ & $0.04^{a}$ & 11.3 & $27.1 \pm 7.1$ & $26.9 \pm 7.4$ & 0.60 & $3 \cdot 3$ \\
\hline Sodium, mmol/L & $138.0 \pm 4.3$ & $138.0 \pm 4.4$ & 0.99 & -0.1 & $138.0 \pm 4.2$ & $138.1 \pm 4.6$ & 0.79 & -1.7 \\
\hline Creatinine, mg/dL & $1.41 \pm 1.5$ & $1.54 \pm 1.4$ & 0.14 & -8.1 & $1.44 \pm 1.6$ & $1.49 \pm 1.5$ & 0.58 & $-3 \cdot 4$ \\
\hline Hemoglobin, g/dL & $12.9 \pm 2.3$ & $13.0 \pm 2.2$ & 0.55 & $-3 \cdot 3$ & $12.9 \pm 2.3$ & $13.0 \pm 2.2$ & 0.82 & -1.4 \\
\hline
\end{tabular}

Values are presented as mean $\pm \mathrm{SD}$ or number $(\%)$.

SMD, standardized mean difference; BMI, body mass index; HF, heart failure; ICMP, ischemic cardiomyopathy; NYHA, New York Heart Association; SBP, systolic blood pressure; DBP, diastolic blood pressure; LVEF, left ventricular ejection fraction.

${ }^{\text {a }}$ Statistically significance.

rate according to the Modification of Diet in Renal Disease equation $[17]$. The primary outcome was all-cause death during follow-up.

\section{Statistical analysis}

Continuous variables were compared using the $t$ test. Categorical variables were compared using the chisquare test and presented as numbers and relative frequencies. Survival curves were assessed with Kaplan-Meier analyses, and the significance level was evaluated with the log-rank test. In order to compare the risk of mortality between carvedilol and bisoprolol groups, the Cox proportional hazard regression was used to calculate the hazard ratio (HR) and 95\% confidence interval (CI). In multivariable models, variables found to be either statistically significant on univariate analysis or clinically relevant were considered candidate variables.

Propensity score matching analyses were performed to reduce the possibility of biased effect estimates in the observational studies. A full, nonparsimonious model was developed. All of the included variables are shown in Tables 1 and 2. Patients in both groups (carvedilol vs. bisoprolol) were matched 1:1 based on the logit of the propensity score with a caliper of width 0.20 of the standard deviation of the logit of the propensity score. The covariate balance achieved by matching was assessed by calculating the absolute standardized mean differences, as listed in Tables 1 and 2. The absolute differences of all variables between the two groups were 
Table 2. Treatment strategy and outcomes on admission

\begin{tabular}{|c|c|c|c|c|c|c|c|c|}
\hline \multirow[b]{2}{*}{ Characteristic } & \multicolumn{4}{|c|}{ Overall population } & \multicolumn{4}{|c|}{ Propensity matched population } \\
\hline & $\begin{array}{l}\text { Bisoprolol } \\
(\mathrm{n}=553)\end{array}$ & $\begin{array}{l}\text { Carvedilol } \\
(\mathrm{n}=831)\end{array}$ & $p$ value & SMD & $\begin{array}{l}\text { Bisoprolol } \\
(\mathrm{n}=508)\end{array}$ & $\begin{array}{l}\text { Carvedilol } \\
(\mathrm{n}=508)\end{array}$ & $p$ value & SMD \\
\hline \multicolumn{9}{|l|}{ Treatments } \\
\hline BB dose, mg & $2.50(1.25-2.50)$ & $6.25(6.25-12.5)$ & NA & NA & $2.50(1.25-2.50)$ & $6.25(6.25-12.5)$ & NA & NA \\
\hline $\mathrm{BB}$ dose, $\mathrm{mg}$ & $2.3 \pm 1.6$ & $11.5 \pm 8.9$ & NA & NA & $2.3 \pm 1.5$ & $11.2 \pm 8.6$ & NA & NA \\
\hline BB dose equivalent, $\%$ & $23 \cdot 3 \pm 15 \cdot 9$ & $23.0 \pm 17.7$ & 0.72 & 1.9 & $23.1 \pm 15.5$ & $22.4 \pm 17.2$ & 0.48 & $4 \cdot 4$ \\
\hline AAs at discharge & $268(48.5)$ & $509(61.3)$ & $<0.001^{\mathrm{a}}$ & -25.9 & $257(50.6)$ & $260(51.2)$ & 0.90 & -1.2 \\
\hline RASB at discharge & $461(83.4)$ & $694(83.5)$ & $>0.99$ & -0.4 & $423(83 \cdot 3)$ & $427(84.1)$ & 0.80 & -2.1 \\
\hline Loop diuretics & $526(95.1)$ & $786(94 \cdot 6)$ & 0.75 & 2.4 & $484(95 \cdot 3)$ & $479(94 \cdot 3)$ & 0.57 & $4 \cdot 6$ \\
\hline Intravenous inotropes & $163(29.5)$ & $210(25 \cdot 3)$ & 0.10 & $9 \cdot 4$ & $151(29.7)$ & $152(29.9)$ & $>0.99$ & -0.4 \\
\hline Transfusion & $74(13.4)$ & $115(13.8)$ & 0.87 & -1.3 & $69(13.6)$ & $72(14.2)$ & 0.86 & -1.7 \\
\hline Mechanical ventilation & $56(10.1)$ & $90(10.8)$ & 0.74 & $-2 \cdot 3$ & $53(10.4)$ & $55(10.8)$ & 0.92 & -1.3 \\
\hline $\begin{array}{l}\text { Renal replacement } \\
\text { therapy }\end{array}$ & $28(5.1)$ & $57(6.9)$ & 0.21 & 7.6 & $28(5 \cdot 5)$ & $34(6.7)$ & 0.51 & $-5 \cdot 3$ \\
\hline Assist device ${ }^{\mathrm{b}}$ & $28(5.1)$ & $46(5 \cdot 5)$ & 0.79 & 2.1 & $27(5 \cdot 3)$ & $28(5 \cdot 5)$ & $>0.99$ & -0.9 \\
\hline \multicolumn{9}{|l|}{ Outcomes on admission } \\
\hline ICU admission & $272(49.2)$ & $368(44 \cdot 3)$ & 0.08 & 9.8 & $247(48.6)$ & $235(46.3)$ & 0.49 & 4.9 \\
\hline Length of stay, day & $12.8 \pm 14.1$ & $11.6 \pm 13.1$ & 0.09 & 9.2 & $12.7 \pm 14.4$ & $12.0 \pm 15.2$ & 0.42 & $5 \cdot 3$ \\
\hline $\begin{array}{l}\text { SBP at discharge, } \\
\mathrm{mmHg}\end{array}$ & $111.3 \pm 16.7$ & $114.7 \pm 17 \cdot 4$ & $<0.001^{\mathrm{a}}$ & -19.7 & $112.0 \pm 16.9$ & $112.3 \pm 16.9$ & 0.80 & -1.6 \\
\hline
\end{tabular}

Values are presented as median (interquartile range), mean $\pm \mathrm{SD}$, or number (\%).

SMD, standardized mean difference; BB, $\beta$-blocker; NA, not available; AA, aldosterone antagonist; RASB, renin angiotensin system blocker; ICU, intensive care unit; SBP, systolic blood pressure.

${ }^{\text {a }}$ Statistically significance.

${ }^{\mathrm{b}}$ Assist devices included intra-aortic balloon pumps, left ventricular assist devices, extracorporeal membrane oxygenation, and cardiac resynchronization therapy.

within $10 \%$ and not statistically different $(p \geq 0.05)$. Cox proportional hazard models were used to compare the outcomes in the matched groups. Statistical analyses were performed using R Statistical Software version 3.3.1 (R Foundation for Statistical Computing, Vienna, Austria). $p$ values $<0.05$ were considered statistically significant.

\section{RESULTS}

\section{Baseline characteristics}

\section{Overall population}

Among the 3,016 patients with HFrEF who survived acute hospitalization, 1,707 (56.6\%) received a BB upon discharge (Supplementary Table 1). Among those prescribed a BB, 60.5\% received carvedilol, while $32.7 \%$ received bisoprolol (Fig. 2). After excluding patients on very low dose BB (standardized dose of carvedilol $\leq 3.125 \mathrm{mg}$ ) or other types of $\mathrm{BB}$, a total of 831 patients on carvedilol and 553 on bisoprolol were enrolled. The baseline clinical and laboratory characteristics according to group are presented in Table 1. The treatment strategy and outcomes of admission data are presented in Table 2. Compared to patients treated with bisoprolol, those treated with carvedilol had a higher prevalence of hypertension and higher incidence of previous HF admission history. Those in the carvedilol group also had a significantly lower LVEF and higher systolic blood pressure at discharge than did those in the bisoprolol group. Aldosterone antagonists (AAs) were 


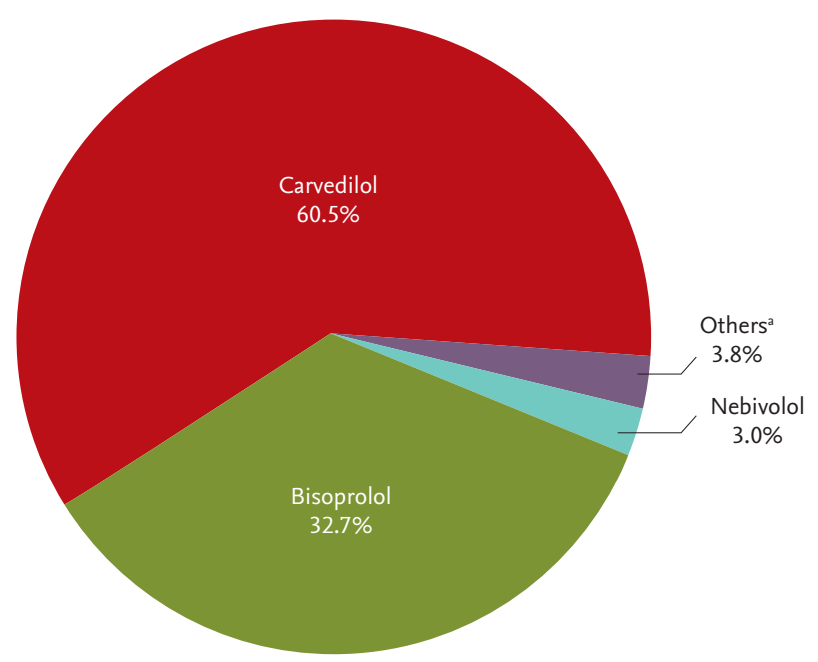

Figure 2. Distribution of $\beta$-blocker (BB) use at discharge. ${ }^{\mathrm{a}}$ Other BBs included metoprolol, betaxolol, celiprolol, atenolol, propranolol, amosulalol, bevantolol, and sotalol.

more frequently prescribed to patients in the carvedilol group than they were to those in the bisoprolol group. The mean daily doses of prescribed carvedilol and bisoprolol were $11.2 \pm 8.6$ and $2.3 \pm 1.6 \mathrm{mg}$, respectively. There was no significant difference between the two groups with regard to the percentage receiving the respective guideline-recommended target doses (Table 2).

\section{Propensity matched population}

After performing propensity score matching, a total of 508 matched patient pairs from the carvedilol and bisoprolol groups were obtained (Tables 1 and 2). In the propensity matched population, there were no significant differences between the groups with regard to the baseline clinical information, laboratory data, treatment strategy, or outcomes from admission data with acceptable standardized mean differences.

\section{Clinical outcomes}

\section{Overall population}

The median follow-up duration was 28 months (interquartile range, 18 to 37). There was a significantly lower risk of mortality in patients who were prescribed a $\mathrm{BB}$ at discharge compared to those who were not (BB vs. no BB, $26.1 \%$ vs. $40.8 \%$; HR, 0.59 ; $95 \%$ CI, 0.52 to 0.67 ; $p$ $<0.001$ ) (Fig. 3). However, there was no significant difference in the rate of all-cause mortality between the

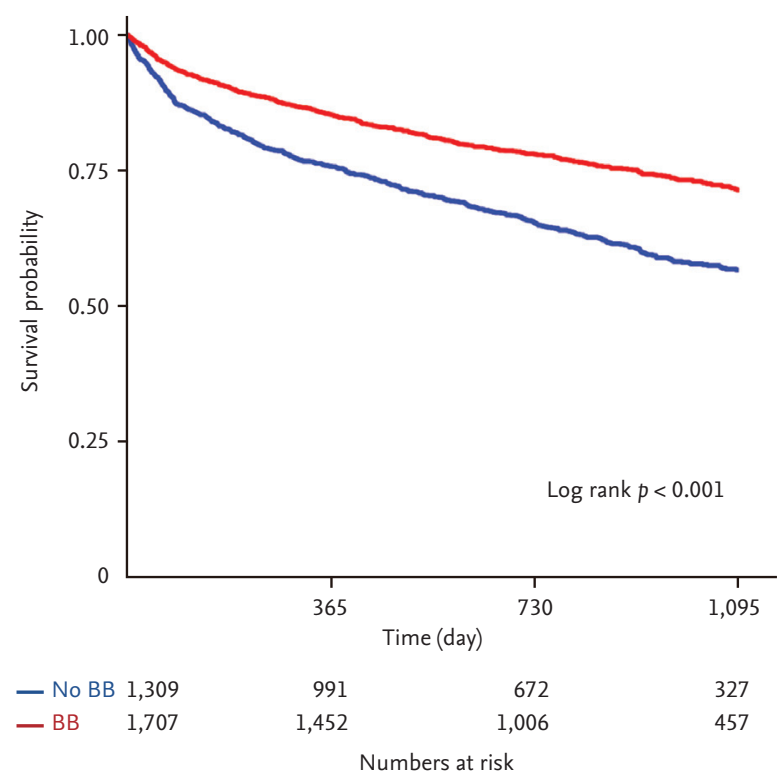

Figure 3. Kaplan-Meier curves for all-cause mortality in acute heart failure (AHF) with heart failure with reduced ejection fraction (HFrEF) according to $\beta$-blocker (BB) use at discharge. Kaplan-Meier curves for all-cause mortality of AHF patients with HFrEF according to the use of BB (red line) or no BB (blue line).

carvedilol and bisoprolol groups (carvedilol vs. bisoprolol, $27.5 \%$ vs. $23.5 \%$; HR, 1.21; $95 \%$ CI, 0.99 to $1.47 ; p=0.07$ ) (Table 3 and Fig. 4A). There was no significant difference in the all-cause death rate between the two groups after adjusting for age, sex, hypertension, diabetes mellitus, history of HF admission, ischemic etiology, systolic blood pressure at discharge, LVEF, renin angiotensin system blocker at discharge, or AA at discharge (adjusted HR, 1.22; 95\% CI, 0.98 to 1.52; $p=0.07$ ) (Table 3).

Propensity matched population

After 1:1 propensity score matching, the bisoprolol and carvedilol groups had a similar risk of all-cause mortality (26.2\% vs. $23.8 \%$; HR, 1.10 ; $95 \%$ CI, 0.86 to $1.40 ; p=$ 0.47) (Table 3 and Fig. 4B).

\section{Subgroup analysis}

Subgroup analysis was performed to evaluate the consistency of the all-cause mortality rate between patients treated with carvedilol and bisoprolol in the propensity-matched population (Fig. 5). The risk of all-cause mortality was similarly observed in various subgroups 
Table 3. All-cause mortality of acute heart failure patients with heart failure with reduced ejection fraction according to $\beta$-blocker type at discharge in total population and propensity-matched population

\begin{tabular}{|c|c|c|c|c|c|c|}
\hline & \multirow{2}{*}{ Bisoprolol } & \multirow{2}{*}{ Carvedilol } & \multicolumn{2}{|c|}{ Unadjusted } & \multicolumn{2}{|c|}{ Adjusted $^{\mathrm{a}}$} \\
\hline & & & $\operatorname{HR}(95 \% \mathrm{CI})$ & $p$ value & $\operatorname{HR}(95 \% \mathrm{CI})$ & $p$ value \\
\hline Overall population & 553 & 831 & & & & \\
\hline All-cause death & $146(23 \cdot 5)$ & $299(27.5)$ & $1.21(0.99-1.47)$ & 0.07 & $1.22(0.98-1.52)$ & 0.07 \\
\hline Propensity matched population & 508 & 508 & & & & \\
\hline All-cause death & $121(23.8)$ & $133(26.2)$ & $1.10(0.86-1.40)$ & 0.47 & $1.19(0.93-1.53)$ & 0.17 \\
\hline
\end{tabular}

Values are presented as number (\%).

$\mathrm{HR}$, hazard ratio; CI, confidence interval.

${ }^{a}$ Adjusted variables included age, male sex, hypertension, diabetes mellitus, history of heart failure admission, ischemic etiology, systolic blood pressure at discharge, left ventricular ejection fraction, renin angiotensin system blocker at discharge, and aldosterone antagonist at discharge.

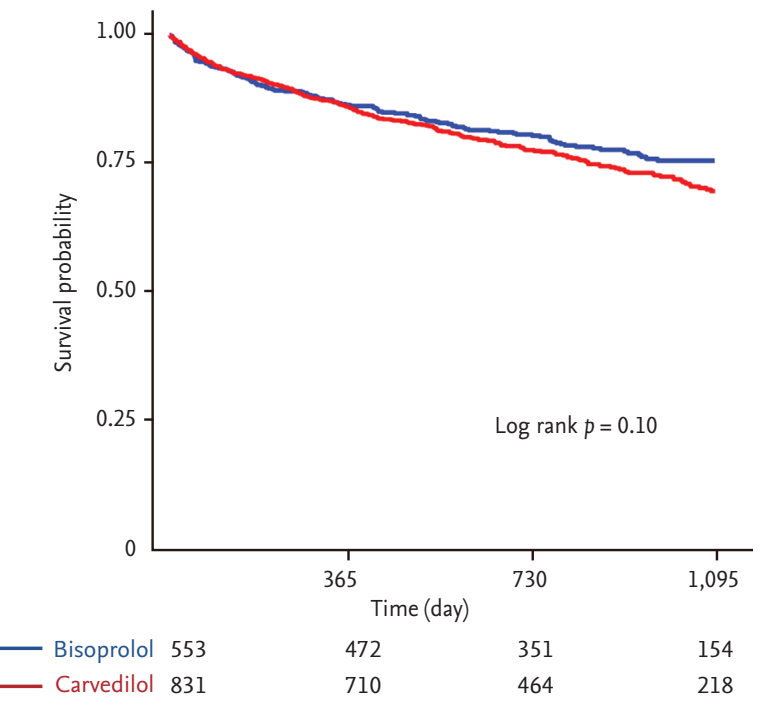

A

Numbers at risk

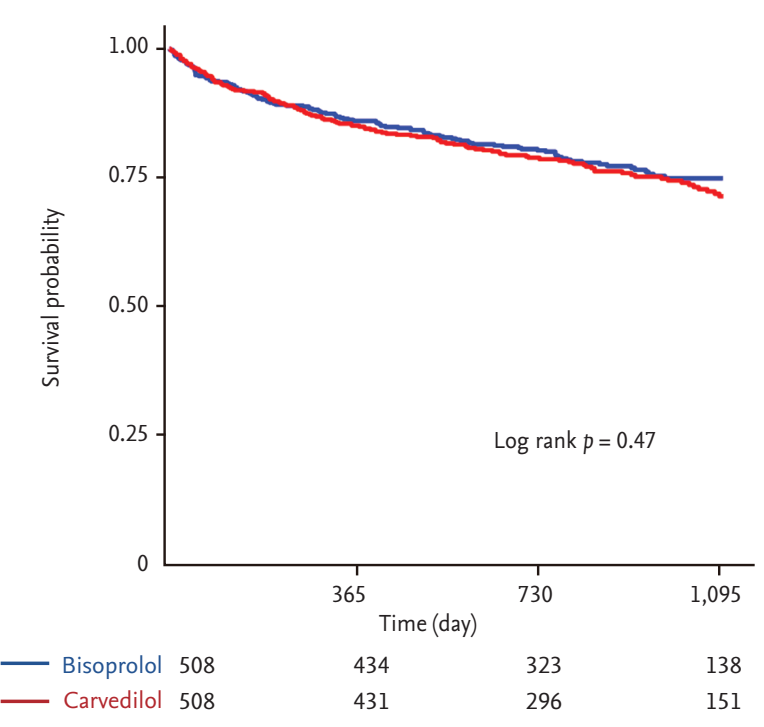

B

Numbers at risk

Figure 4. Comparison of all-cause mortality rate between treatment with carvedilol or bisoprolol. Kaplan-Meier curves for allcause mortality of acute heart failure patients with heart failure with reduced ejection fraction in the overall population (A) and propensity-matched population (B) according to use of carvedilol (red line) or bisoprolol (blue line).

according to patient characteristics without significant interaction.

\section{$\beta$-Blocker duration and dose}

Among the study population, 203 patients (14.7\%) died or were not able to follow-up within 1 year. Among patients with HFrEF who survived more than 1 year after hospitalization, BB was maintained at 1-year follow-up in 862 patients (73.0\%). There was no significant difference in proportion of maintenance of $\mathrm{BB}$ between the carvedilol and bisoprolol groups (carvedilol vs. bisopro- lol, $71.7 \%$ [509/710] vs. $74.9 \%[353 / 771], p=0.23)$. BB dose at 1-year follow-up was increased compared to initial dose according to the current guidelines in both groups, and the follow-up dose equivalent was not significantly different between the two groups (Supplementary Fig. 1).

\section{DISCUSSION}

In this large contemporary cohort study in Korea, there was no significant difference in all-cause mortality 


\begin{tabular}{|c|c|c|c|c|}
\hline Subgroups & No. of patients & Hazard ratio $(95 \% \mathrm{Cl})$ & Hazard ratio $(95 \% \mathrm{Cl})$ & Interaction $\boldsymbol{p}$ value \\
\hline \multicolumn{5}{|l|}{ Age } \\
\hline$>65$ & 559 & & 1.31 (0.98 to 1.73$)$ & \multirow{2}{*}{0.15} \\
\hline$\leq 65$ & 457 & & 0.85 (0.51 to 1.41$)$ & \\
\hline \multicolumn{5}{|l|}{ Sex } \\
\hline Male & 610 & & $1.18(0.85$ to 1.65$)$ & \multirow{2}{*}{0.53} \\
\hline Female & 406 & & $1.01(0.70$ to 1.47$)$ & \\
\hline \multicolumn{5}{|l|}{ Hypertension } \\
\hline Yes & 545 & & $1.09(0.80$ to 1.47$)$ & \multirow{2}{*}{0.82} \\
\hline No & 471 & & 1.15 (0.75 to 1.77$)$ & \\
\hline \multicolumn{5}{|c|}{ Diabetes mellitus } \\
\hline Yes & 397 & & $1.24(0.88$ to 1.75$)$ & \multirow[b]{2}{*}{0.34} \\
\hline No & 619 & & 0.97 (0.68 to 1.39$)$ & \\
\hline \multicolumn{5}{|c|}{ Ischemic etiology } \\
\hline Yes & 389 & & $1.23(0.87$ to 1.73$)$ & \multirow{2}{*}{0.40} \\
\hline No & 627 & & $1.00(0.70$ to 1.42$)$ & \\
\hline \multicolumn{5}{|c|}{ Target dose achievement } \\
\hline Yes & 74 & & $0.67(0.15$ to 2.94$)$ & \multirow{2}{*}{0.51} \\
\hline No & 942 & & 1.11 (0.86 to 1.43$)$ & \\
\hline \multicolumn{5}{|c|}{ Estimated GFR } \\
\hline$\leq 60$ & 472 & & $1.12(0.82$ to 1.51$)$ & \multirow{2}{*}{0.86} \\
\hline$>60$ & 544 & & $1.07(0.70$ to 1.62$)$ & \\
\hline \multicolumn{5}{|c|}{ Vasopressor use } \\
\hline Yes & 303 & & $0.97(0.63$ to 1.48$)$ & \multirow{2}{*}{0.51} \\
\hline No & 713 & & $1.17(0.86$ to 1.58$)$ & \\
\hline \multicolumn{5}{|c|}{ Atrial fibrillation } \\
\hline Yes & 214 & & $1.08(0.62$ to 1.88$)$ & \multirow{2}{*}{0.96} \\
\hline No & 802 & & $1.10(0.83$ to 1.45$)$ & \\
\hline \multicolumn{5}{|c|}{ Systolic blood pressure $<100 \mathrm{mmHg}$} \\
\hline Yes & 114 & & $1.12(0.55$ to 2.28$)$ & \multirow{2}{*}{0.73} \\
\hline & 902 & & 1.05 (0.81 to 1.38$)$ & \\
\hline \multicolumn{5}{|c|}{ Heart rate $>100 \mathrm{bpm}$} \\
\hline Yes & 367 & & 0.93 (0.60 to 1.45$)$ & \multirow{2}{*}{0.36} \\
\hline No & 649 & & $1.19(0.89$ to 1.61$)$ & \\
\hline \multicolumn{5}{|c|}{ Ejection fraction $<25 \%$} \\
\hline Yes & 385 & & $1.44(0.94$ to 2.19$)$ & \multirow{2}{*}{0.11} \\
\hline \multirow[t]{3}{*}{ No } & 631 & & 0.94 (0.69 to 1.28$)$ & \\
\hline & & & & \\
\hline & & & & \\
\hline
\end{tabular}

Figure 5. Subgroup analysis. Comparative unadjusted hazard ratios of all-cause mortality for subgroups in the propensity matched population between carvedilol and bisoprolol groups. CI, confidence interval; GFR, glomerular filtration rate.

between carvedilol and bisoprolol at discharge in hospitalized patients with AHF and HFrEF. The primary finding was consistent after performing propensity score matching in an effort to reduce selection bias. In particular, the effects of carvedilol and bisoprolol were consistent through various subgroups of patients. These subgroups included patients with ischemic etiology of their HF, target dose users, those with or without vasopressor requirements on admission, and those with atrial fibrillation and renal dysfunction. Our study also shows that, regardless of the type of BB used, there is a consistent mortality benefit in patients with AHF and HFrEF.

Carvedilol is a third-generation BB with competitive blockade of the $\beta 1-, \beta 2-$, and $\alpha 1$-adrenergic receptors. Among all BBs, carvedilol has been studied most extensively in patients with HF [5,6,18-20]. Therefore, some investigators have suggested that, given its vasodilatory effect, carvedilol would have more robust clinical outcomes in HFrEF than selective $\beta 1$ inhibitors, including bisoprolol and metoprolol. The COMET (Carvedilol Or Metoprolol European Trial) study, a large randomized controlled trial comparing carvedilol and metoprolol, found that carvedilol extended survival compared to metoprolol in patients with chronic HF [8]. However, this study had several critical limitations. For one, carvedilol was compared to short-acting metoprolol tartrate. However, metoprolol tartrate has not been 
shown to have a mortality benefit in HF and is therefore not recommended in the practice guidelines. In addition, the group used a dose of metoprolol that is lower than the recommended guideline doses; in contrast, carvedilol was prescribed at full guideline target dose. Another randomized trial that compared outcomes between different BB types was the MAINCHF II study, which was a head-to-head comparison of bisoprolol and carvedilol in Japanese patients with chronic HF [21]. Unfortunately, this study was terminated early because bisoprolol was approved for use in the treatment of chronic HF. Given the limitations of these previous randomized trials, many observational studies and meta-analyses have been performed in an effort to compare different BBs in the treatment of HF. However, the results of these studies are controversial [9-11,22].

The KorAHF registry included patients from 10 tertiary care centers throughout Korea, and enrolled a relatively large number of HF patients. Fortunately, this registry provided a large number of clinical and laboratory variables, as well as many well-known prognostic factors. Consequently, we were able to perform propensity-matching analysis to adjust for these prognostic factors. The difference in mortality benefit between carvedilol and bisoprolol was not significant prior to matching and became even more negligible in the matched cohort. Our results and those of previous studies suggest that carvedilol and bisoprolol have comparable mortality benefits in patients with HFrEF. The current study also showed that baseline characteristics of the study population according to use of carvedilol or bisoprolol were slightly different. Patients prescribed carvedilol had a higher proportion of hypertension, pervious history of HF admission, and lower EF compared to those with bisoprolol. We postulated that this might be caused by more variety in dosage of carvedilol compared to bisoprolol, although the selection of type of BB was based on the physician's preference. However, initial treatment dose equivalent, 1-year maintenance rates, and doses were similar between the carvedilol and bisoprolol groups. This would support that both carvedilol and bisoprolol are well-tolerated in hospitalized patients with AHF and HFrEF after stabilization.

Konishi et al. [23] found that 107 patients with severe congestive $\mathrm{HF}$ on bisoprolol and 110 patients on carvedilol had similar clinical outcomes. However, the group also found that bisoprolol had greater benefits in patients with $\mathrm{HF}$ and concurrent atrial fibrillation than did similar patients taking carvedilol. In contrast to the previous study, we did not identify any significant interactions between BB type and various patient subgroups, including those with atrial fibrillation. In the future, a large randomized controlled trial is needed to substantiate our findings and identify any differences between carvedilol and bisoprolol in this specific population.

With regard to AHF, many physicians are concerned with the initiation or maintenance of BBs given their negative inotropic properties, which can exacerbate organ hypoperfusion and fluid congestion. However, in prior studies, use of BB during hospitalization for $\mathrm{HF}$ was actually associated with a significant reduction in the rate of death or re-hospitalization due to HF [24-26]. Furthermore, the IMPACT-HF trial found that pre-discharge use of carvedilol improved the use of BB at 60 days without an increase in side effects or length of stay in hospitalized patients with acute decompensated HF [27]. The OPTIMIZE-HF trial also showed that treatment with carvedilol or another evidence-based BB at discharge was well-tolerated and reduced mortality risk compared to that in patients who were not prescribed a BB on discharge [20]. In this study, we found a similar mortality benefit with BB use upon discharge of patients hospitalized for AHF. In addition, our data showed that there was no significant difference in the all-cause mortality benefit between treatment with carvedilol and bisoprolol in patients with AHF. These results suggest that a $\mathrm{BB}$ ought to be initiated after stabilization of AHF during hospitalization, as stated in the current guidelines $[1,2]$. Furthermore, our findings suggest that the type of $\mathrm{BB}$, at least between carvedilol or bisoprolol, does not affect the mortality benefit.

This study has several limitations. First, the KorAHF registry was not a randomized controlled trial. Therefore, the specific types of BBs used may have reflected individual physician preferences. We also did not have information regarding the reasons why physicians did not prescribe a BB to some patients at discharge after stabilization of their AHF. A future well-designed randomized controlled trial for comparing carvedilol and bisoprolol would be helpful to confirm our study. 
A second limitation is that, although we performed risk adjustments for potential confounding factors, including propensity score matching, we were unable to adjust for unmeasured variables. Third, because most participating centers in this study were large tertiary hospitals in Korea, patients with de novo HF were more frequently included compared to the other HF registry data. In this regard, relatively low rates of mortality may be observed, and our results cannot be extrapolated to patients admitted to primary care hospitals with small volume. Finally, this study was not adequately powered to assess outcome differences among other evidence-based BBs, including metoprolol and nebivolol, because of their low prescription rate.

In the treatment of AHF with reduced EF after hospitalization, patients prescribed a BB upon discharge had significantly lower risk of all-cause mortality compared to those who were not prescribed a BB. The mortality benefits of carvedilol and bisoprolol were comparable in AHF patients with HFrEF.

\section{KEY MESSAGE}

1. In the treatment of acute heart failure with reduced ejection fraction after hospitalization, use of a $\beta$-blocker at discharge shows a mortality benefit in patients with acute heart failure with reduced ejection fraction.

2. The mortality benefits of carvedilol and bisoprolol, which are widely used and have demonstrated supporting evidence for the treatment of heart failure, are comparable in acute heart failure patients with reduced ejection fraction.

\section{Conflict of interest}

No potential conflict of interest relevant to this article was reported.

\section{Acknowledgments}

This work was supported by the Research of Korea Centers for Disease Control and Prevention (2010-E63003-00, 2011-E63002- 00, 2012-E63005-00, 2013-E63003-00, 201318 E63003-01, 2013- E63003-02, and 2016-ER6303-00).

\section{REFERENCES}

1. Ponikowski P, Voors AA, Anker SD, et al. 2016 ESC guidelines for the diagnosis and treatment of acute and chronic heart failure: the task force for the diagnosis and treatment of acute and chronic heart failure of the European Society of Cardiology (ESC) Developed with the special contribution of the Heart Failure Association (HFA) of the ESC. Eur Heart J 2016;37:2129-2200.

2. Yancy CW, Jessup M, Bozkurt B, et al. 2013 ACCF/AHA guideline for the management of heart failure: a report of the American College of Cardiology Foundation/American Heart Association Task Force on Practice Guidelines. J Am Coll Cardiol 2013;62:e147-e239.

3. MERIT-HF Study Group. Effect of metoprolol CR/XL in chronic heart failure: Metoprolol CR/XL Randomised Intervention Trial in Congestive Heart Failure (MERIT-HF). Lancet 1999;353:2001-2007.

4. CIBIS-II Investigators and Committees. The Cardiac Insufficiency Bisoprolol Study II (CIBIS-II): a randomised trial. Lancet 1999;353:9-13.

5. Packer M, Bristow MR, Cohn JN, et al. The effect of carvedilol on morbidity and mortality in patients with chronic heart failure. U.S. Carvedilol Heart Failure Study Group. N Engl J Med 1996;334:1349-1355.

6. Packer M, Fowler MB, Roecker EB, et al. Effect of carvedilol on the morbidity of patients with severe chronic heart failure: results of the carvedilol prospective randomized cumulative survival (COPERNICUS) study. Circulation 2002;106:2194-2199.

7. Gilbert EM, Abraham WT, Olsen S, et al. Comparative hemodynamic, left ventricular functional, and antiadrenergic effects of chronic treatment with metoprolol versus carvedilol in the failing heart. Circulation 1996;94:28172825.

8. Poole-Wilson PA, Swedberg K, Cleland JG, et al. Comparison of carvedilol and metoprolol on clinical outcomes in patients with chronic heart failure in the Carvedilol Or Metoprolol European Trial (COMET): randomised controlled trial. Lancet 2003;362:7-13.

9. Pasternak B, Svanstrom H, Melbye M, Hviid A. Association of treatment with carvedilol vs metoprolol succinate and mortality in patients with heart failure. JAMA Intern Med 2014;174:1597-1604.

10. Frohlich H, Torres L, Tager T, et al. Bisoprolol compared with carvedilol and metoprolol succinate in the treat- 
ment of patients with chronic heart failure. Clin Res Cardiol 2017;106:711-721.

11. Frohlich H, Zhao J, Tager T, et al. Carvedilol compared with metoprolol succinate in the treatment and prognosis of patients with stable chronic heart failure: carvedilol or metoprolol evaluation study. Circ Heart Fail 2015;8:887896.

12. Packer M, Antonopoulos GV, Berlin JA, Chittams J, Konstam MA, Udelson JE. Comparative effects of carvedilol and metoprolol on left ventricular ejection fraction in heart failure: results of a meta-analysis. Am Heart J 2001;141:899-907.

13. Chatterjee S, Biondi-Zoccai G, Abbate A, et al. Benefits of $\beta$ blockers in patients with heart failure and reduced ejection fraction: network meta-analysis. BMJ 2013;346:f55.

14. Lee SE, Lee HY, Cho HJ, et al. Clinical characteristics and outcome of acute heart failure in Korea: results from the Korean Acute Heart Failure registry (KorAHF). Korean Circ J 2017;47:341-353.

15. Lee SE, Cho HJ, Lee HY, et al. A multicentre cohort study of acute heart failure syndromes in Korea: rationale, design, and interim observations of the Korean Acute Heart Failure (KorAHF) registry. Eur J Heart Fail 2014;16:700708.

16. Schiller NB, Shah PM, Crawford M, et al. Recommendations for quantitation of the left ventricle by two-dimensional echocardiography. American Society of Echocardiography Committee on Standards, Subcommittee on Quantitation of Two-Dimensional Echocardiograms. J Am Soc Echocardiogr 1989;2:358-367.

17. Levey AS, Coresh J, Greene T, et al. Using standardized serum creatinine values in the modification of diet in renal disease study equation for estimating glomerular filtration rate. Ann Intern Med 2006;145:247-254.

18. Hori M, Sasayama S, Kitabatake A, et al. Low-dose carvedilol improves left ventricular function and reduces cardiovascular hospitalization in Japanese patients with chronic heart failure: the Multicenter Carvedilol Heart Failure Dose Assessment (MUCHA) trial. Am Heart J 2004;147:324-330.

19. Di Lenarda A, Sabbadini G, Salvatore L, et al. Long-term effects of carvedilol in idiopathic dilated cardiomyopa- thy with persistent left ventricular dysfunction despite chronic metoprolol. The Heart-Muscle Disease Study Group. J Am Coll Cardiol 1999;33:1926-1934.

20. Fonarow GC, Abraham WT, Albert NM, et al. Carvedilol use at discharge in patients hospitalized for heart failure is associated with improved survival: an analysis from Organized Program to Initiate Lifesaving Treatment in Hospitalized Patients with Heart Failure (OPTIMIZE-HF). Am Heart J 2007;153:82.

21. Hori M, Nagai R, Izumi T, Matsuzaki M. Efficacy and safety of bisoprolol fumarate compared with carvedilol in Japanese patients with chronic heart failure: results of the randomized, controlled, double-blind, Multistep Administration of bisoprolol IN Chronic Heart Failure II (MAIN-CHF II) study. Heart Vessels 2014;29:238-247.

22. Bolling R, Scheller NM, Kober L, Poulsen HE, Gislason GH, Torp-Pedersen C. Comparison of the clinical outcome of different beta-blockers in heart failure patients: a retrospective nationwide cohort study. Eur J Heart Fail 2014;16:678-684.

23. Konishi M, Haraguchi G, Kimura S, et al. Comparative effects of carvedilol vs bisoprolol for severe congestive heart failure. Circ J 2010;74:1127-1134.

24. Butler J, Young JB, Abraham WT, et al. Beta-blocker use and outcomes among hospitalized heart failure patients. J Am Coll Cardiol 2006;47:2462-2469.

25. Metra M, Torp-Pedersen C, Cleland JG, et al. Should beta-blocker therapy be reduced or withdrawn after an episode of decompensated heart failure? Results from COMET. Eur J Heart Fail 2007;9:901-909.

26. Fonarow GC, Abraham WT, Albert NM, et al. Influence of beta-blocker continuation or withdrawal on outcomes in patients hospitalized with heart failure: findings from the OPTIMIZE-HF program. J Am Coll Cardiol 2008;52:190199.

27. Gattis WA, O'Connor CM, Gallup DS, Hasselblad V, Gheorghiade M; IMPACT-HF Investigators and Coordinators. Predischarge initiation of carvedilol in patients hospitalized for decompensated heart failure: results of the Initiation Management Predischarge: Process for Assessment of Carvedilol Therapy in Heart Failure (IMPACT-HF) trial. J Am Coll Cardiol 2004;43:1534-1541. 
Supplementary Table 1. Baseline clinical, laboratory characteristics, treatment strategy, and outcomes on admission according to prescription of $\beta$-blocker

\begin{tabular}{|c|c|c|c|}
\hline Characteristic & $\mathrm{BB}(\mathrm{n}=1,707)$ & No BB $(n=1,309)$ & $p$ value \\
\hline Age, yr & $64.96 \pm 14.7$ & $67.34 \pm 14.9$ & $<0.001^{\mathrm{a}}$ \\
\hline Male sex & $1,036(60.7)$ & $809(61.8)$ & 0.560 \\
\hline Current smoker & $405(23.7)$ & $248(18.9)$ & $0.002^{\mathrm{a}}$ \\
\hline Body mass index, $\mathrm{kg} / \mathrm{m}^{2}$ & $23.5 \pm 3.9$ & $22.8 \pm 3.8$ & $<0.001^{\mathrm{a}}$ \\
\hline Hypertension & $958(56.1)$ & $703(53 \cdot 7)$ & 0.199 \\
\hline Diabetes mellitus & $644(37 \cdot 7)$ & $466(35 \cdot 6)$ & 0.245 \\
\hline Chronic kidney disease & $214(12.5)$ & $212(16.2)$ & $0.005^{\mathrm{a}}$ \\
\hline Pulmonary disease & $137(8.0)$ & $174(13 \cdot 3)$ & $<0.001^{\mathrm{a}}$ \\
\hline Prior HF admission & $483(28.3)$ & $513(39.2)$ & $<0.001^{\mathrm{a}}$ \\
\hline De novo HF & $977(57 \cdot 2)$ & $576(44.0)$ & $<0.001^{\mathrm{a}}$ \\
\hline ICMP & $696(40.8)$ & $551(42.1)$ & 0.489 \\
\hline Atrial fibrillation & $507(29.7)$ & $367(28.0)$ & 0.338 \\
\hline $\mathrm{NYHA} \geq 3$ & $1,461(85 \cdot 6)$ & $1,136(86.8)$ & 0.375 \\
\hline $\mathrm{SBP}, \mathrm{mmHg}$ & $131.5 \pm 29.1$ & $125.2 \pm 28.2$ & $<0.001^{\mathrm{a}}$ \\
\hline $\mathrm{DBP}, \mathrm{mmHg}$ & $81.9 \pm 18.9$ & $76.9 \pm 17.6$ & $<0.001^{\mathrm{a}}$ \\
\hline Pulse rate, beats/min & $95 \cdot 7 \pm 24 \cdot 6$ & $95 \cdot 5 \pm 25 \cdot 6$ & 0.809 \\
\hline LVEF, \% & $26.7 \pm 7.4$ & $26.8 \pm 7.5$ & 0.758 \\
\hline Sodium, mmol/L & $137.9 \pm 4.4$ & $137.1 \pm 4.9$ & $<0.001^{\mathrm{a}}$ \\
\hline Creatinine, mg/dL & $1.47 \pm 1.4$ & $1.52 \pm 1.5$ & 0.333 \\
\hline Hemoglobin, g/dL & $12.9 \pm 2.3$ & $12.6 \pm 2.3$ & $<0.001^{\mathrm{a}}$ \\
\hline AAs at discharge & $970(56.8)$ & $610(46.6)$ & $<0.001^{\mathrm{a}}$ \\
\hline RASB at discharge & $1,408(82.5)$ & $885(67.6)$ & $<0.001^{\mathrm{a}}$ \\
\hline Loop diuretics & $1,615(94 \cdot 6)$ & $1,232(94.1)$ & 0.615 \\
\hline Intravenous inotropes & $470(27.5)$ & $562(42.9)$ & $<0.001^{\mathrm{a}}$ \\
\hline Transfusion & $238(13.9)$ & $280(21.4)$ & $<0.001^{\mathrm{a}}$ \\
\hline Mechanical ventilation & $186(10.9)$ & $215(16.4)$ & $<0.001^{\mathrm{a}}$ \\
\hline Renal replacement therapy & $99(5.8)$ & $89(6.8)$ & 0.294 \\
\hline Assist device ${ }^{\mathrm{b}}$ & $89(5.2)$ & $83(6.3)$ & 0.214 \\
\hline ICU admission & $786(46.0)$ & $671(51.3)$ & $0.005^{\mathrm{a}}$ \\
\hline Length of stay, day & $12.1 \pm 13.8$ & $14.8 \pm 18.4$ & $<0.001^{\mathrm{a}}$ \\
\hline $\mathrm{SBP}$ at discharge, $\mathrm{mmHg}$ & $113 \cdot 3 \pm 16.9$ & $111.9 \pm 16.9$ & $0.025^{\mathrm{a}}$ \\
\hline
\end{tabular}

Values are presented as mean \pm SD or number (\%).

BB, $\beta$-blocker; HF, heart failure; ICMP, ischemic cardiomyopathy; NYHA, New York Heart Association; SBP, systolic blood pressure; DBP, diastolic blood pressure; LVEF, left ventricular ejection fraction; AA, aldosterone antagonist; RASB, renin angiotensin system blocker; ICU, intensive care unit.

${ }^{\mathrm{a}}$ Statistically significance.

${ }^{\mathrm{b}}$ Assist devices included intra-aortic balloon pumps, left ventricular assist devices, extracorporeal membrane oxygenation, and cardiac resynchronization therapy. 


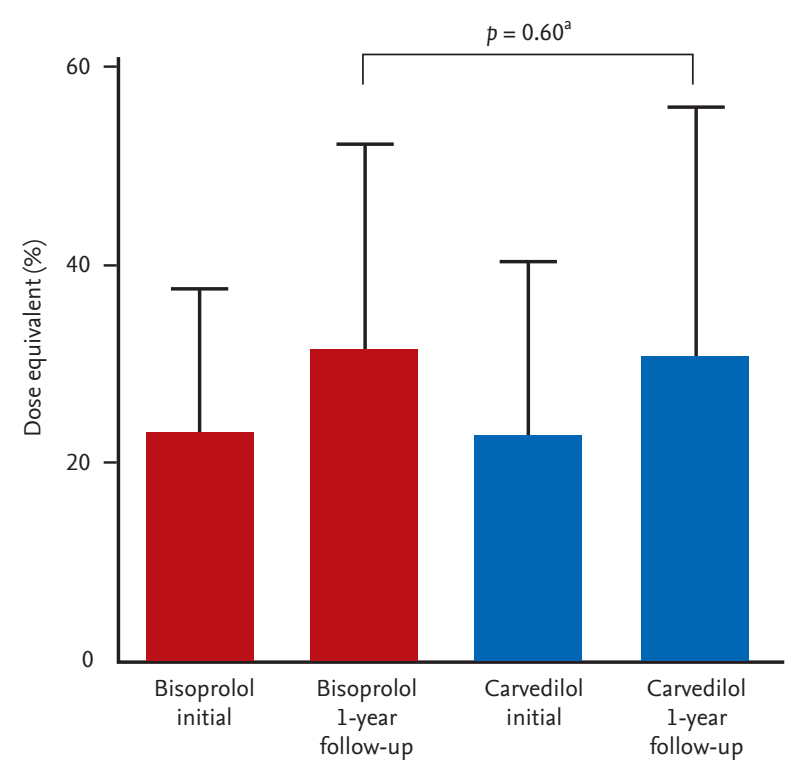

Supplementary Figure 1. Initial and 1-year follow-up $\beta$-blocker dose equivalent according to carvedilol and bisoprolol. Orange bars denotes bisoprolol and blue bars denotes carvedilol. ap value for comparison between 1-year follow-up dose equivalent between bisoprolol and carvedilol. 